\title{
Reappraisal of Left Ventricular to Right Atrial (LV-RA) Shunt in Pediatric Patients With Isolated Perimembranous Ventricular Septal Defect
}

\author{
Ken-Pen Weng, MD*,†,I; Shi-Hui Huang, MS**,I; Chu-Chuan Lin, MD*; \\ Shih-Ming Huang, MD ${ }^{\dagger+}$; Kuang-Jen Chien, MD*; \\ Luo-Ping Ger, MPH*; Kai-Sheng Hsieh, MD*
}

\begin{abstract}
Background The purpose of this study was to investigate the follow-up results of perimembranous ventricular septal defect (VSD) with left ventricular to right atrial (LV-RA) shunt since infancy and to analyze the morphologic variations of this shunt.

Methods and Results The study group comprised 232 consecutive pediatric patients with isolated perimembranous VSD and aneurysm, of whom 134 (58\%) had LV-RA shunts. Follow-up echocardiography was performed to assess for the size of both the VSD and LV-RA shunt. There were no significant differences between groups in terms of sex, age at the initial echocardiography, follow-up period, number of patients with tricuspid regurgitation, and initial VSD size. There was a significant difference between groups in spontaneous closure ( $\mathrm{p}=0.039)$. The event-free probability (no surgical repair of the defect) was not significantly different between the groups $(\mathrm{p}=0.129)$.

Conclusions Perimembranous VSD with LV-RA shunt in infancy is common and associated with less chance of spontaneous closure. Color Doppler echocardiography can greatly improves the diagnostic efficacy and assist in understanding the mechanisms leading to this particular anomaly. (Circ J 2008; 72: 1487-1491)
\end{abstract}

Key Words: Aneurysm; Left ventricular to right atrial shunt; Ventricular septal defect

I n patients with perimembranous ventricular septal defect (VSD), a left ventricular to right atrial (LV-RA) is associated with aneurysm formation and anomalies of the tricuspid valve ${ }^{-5}$ Before the era of echocardiography, correct diagnosis of LV-RA shunt was established only at catheterization or surgery-9 9 The 2-dimensional (D) echocardiography with color Doppler examination has become a reliable, noninvasive technique of establishing the diagnosis of LV-RA shunt. In previous studies color Doppler has revealed 3-15\% of patients with perimembranous VSD and LV-RA shunt?,10-13 However, there are limited details in the literature about the natural history of this anomaly, so this study was designed to investigate the follow-up results of perimembranous VSD with LV-RA shunt since infancy and to analyze the morphologic variations of this shunt.
(Received February 7, 2008; revised manuscript received April 18, 2008; accepted April 23, 2008)

*Department of Pediatrics and Medical Education and Research, Kaohsiung Veterans General Hospital, National Yang-Ming University, **Department of Nursing, Fooyin University, Kaohsiung Medical University, ${ }^{\dagger}$ Department of Pediatrics, Zuoying Armed Forces Hospital and †epartment of Pediatrics, Kaohsiung Municipal United Hospital, Kaohsiung, Taiwan

IThe first two authors contributed equally to this work.

Mailing address: Kai-Sheng Hsieh, MD, Department of Pediatrics and Medical Education and Research, Kaohsiung Veterans General Hospital, 386, Ta-Chung Road, Kaohsiung, Taiwan 813. E-mail: kshsieh@isca.vghks.gov.tw

All rights are reserved to the Japanese Circulation Society. For permissions, please e-mail: cj@j-circ.or.jp

\section{Methods}

\section{Study Design and Subjects}

Between September 2001 and December 2006, 232 consecutive pediatric patients with isolated perimembranous VSD and aneurysm were recruited and received regular follow-up. Of them 134 (58\%) had LV-RA shunts. Follow-up echocardiography was performed to assess the size of both the VSD and LV-RA shunt every 1-3 months during the first year, and then every 3-6 months thereafter. The echocardiographic measurements were performed using a VingMed Sound (GE) machine and the images were assessed inde-

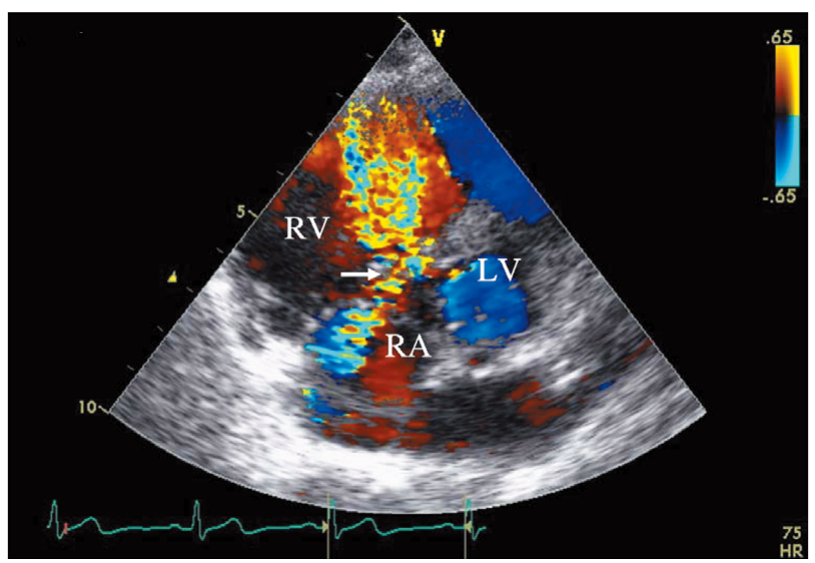

Fig 1. Parasternal short-axis view shows the color flow jet from the left ventricular (LV) to the right atrial (RA), called the bank-shot sign (arrow). RV, right ventricular. 

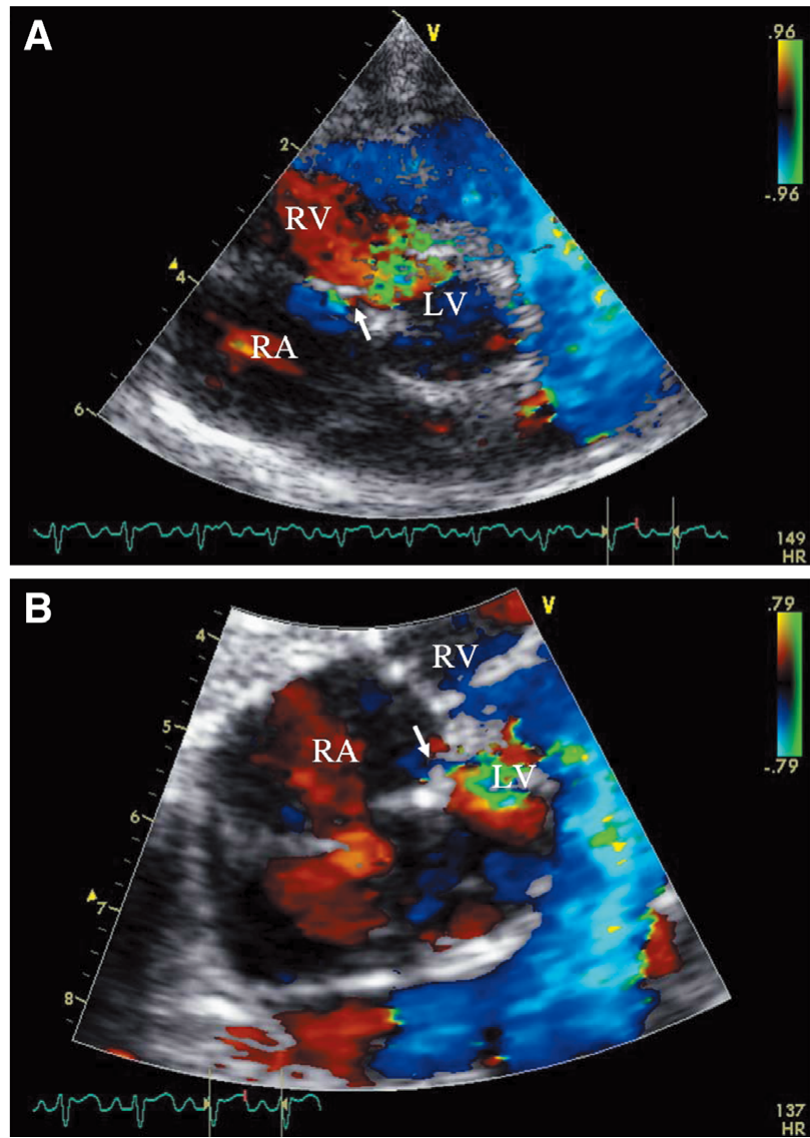

Fig 2. (A) Parasternal short-axis view shows the trivial left ventricular to right atrial (LV-RA) shunt (arrow). (B) Subcostal short-axis view also shows the trivial LV-RA shunt (arrow). LV, left ventricular; $\mathrm{RA}$, right atrial; RV, right ventricular.

pendently by 2 of the authors.

\section{Diagnostic Criteria of Color Doppler Echocardiography}

A perimembranous VSD was diagnosed on the left ventricular (LV) outflow tract short-axis view as an area of discontinuity adjacent to the tricuspid valve leaflet ${ }^{11}$ Aneurysm of the perimembranous VSD was defined as echocardiographic evidence of tissue surrounding the VSD, forming a sac-like structure that protruded into the right ventricle during systole and seemed to partially occlude the flow through the VSD2,11

Diagnosis of LV-RA shunt were based on 2 integrated color Doppler echocardiographic criteria. (1) The presence of a color flow jet from the LV to the right atrial (RA); that is, the "bank-shot sign", which means the curved flow pattern at the LV site of the aneurysm looks like the bankshot in basketball (Fig 1). To avoid false-positive signals, color flow mapping was performed according to a standard sequence ${ }^{14,15}$ In brief, the color gain control was adjusted to the minimum and increased gradually until the tissue echocardiograms began to have background color signals. The gain was finely decreased to the point of exact disappearance of these color signals. Particular attention was paid to the color flow signals in the area of the VSD, the leaflets of the tricuspid valve, and the atrioventricular portion of the interventricular septum. Care was taken to define the particular jet and not to confuse tricuspid regurgitation (TR) with LV-RA shunt. (2) Doppler demonstration of highvelocity turbulence $(>4.0 \mathrm{~m} / \mathrm{s})$ moving from the $\mathrm{LV}$, through the VSD, and directly into the RA2,3 When LV-RA shunt is present, there is a reversed downward flow peak velocity of more than $4.0 \mathrm{~m} / \mathrm{s}$ in the high-pulse repetition-frequency Doppler mode with the sample volume set at the most proximal portion of the RA section of the LV-RA jet. The pressure gradient of the LV-RA shunt is equivalent to the difference between the systolic LV and RA pressures and can be readily differentiated from TR on the basis of the high velocity of the LV-RA shunt in the absence of pulmonary stenosis and pulmonary hypertension. If the diagnosis of LV-RA shunt is based mainly on the bank-shot sign, it is a trivial LV-RA shunt (Figs 2A,B). A small-to-moderate LV-RA shunt is defined as the presence of both criteria.

The 2-D echocardiography was used to the grade the VSD in terms of the size of the aortic root: large $(>2 / 3)$, moderate $(\leq 2 / 3$ and $\geq 1 / 3)$, and mild $(<1 / 3))^{16}$

\section{Follow-up Outcome}

During follow-up, a smaller VSD was defined as a decrease in the size of the VSD and a decrease in the size of the previously enlarged LV and LA on 2-D echocardiography. Spontaneous closure was defined by failure to find color Doppler evidence of a VSD. Indications for surgical repair were uncontrolled congestive heart failure, large defects with elevated pulmonary artery pressure, and Qp/Qs $>2: 1$.

\section{Statistical Analysis}

The descriptions of basic data, such as age and sex, are expressed as the mean $\pm \mathrm{SD}$. Student's t-test was used for comparison of continuous variable data. Differences in categorical variables were evaluated by chi-square test and Fisher's exact test. The advantage of Fisher's exact test was

Table 1 Summary of the Patients' Characteristics

\begin{tabular}{lccc}
\hline \hline & $\begin{array}{c}\text { With LV-RA shunt } \\
(n=134)\end{array}$ & $\begin{array}{c}\text { Without LV-RA shunt } \\
(n=98)\end{array}$ & p value \\
\hline Sex $(M / F)$ & $79 / 55$ & $65 / 33$ & 0.314 \\
Age at initial echo (months) & $3 \pm 3$ & $3 \pm 4$ & 0.586 \\
FU period (months) & $28 \pm 9$ & $26 \pm 9$ & 0.891 \\
No. of patients with TR & 128 & 91 & 0.560 \\
Initial VSD size & & & 0.994 \\
Mild & 95 & 70 & \\
Moderate & 29 & 21 & \\
Severe & 10 & 7 & \\
\hline
\end{tabular}

LV-RA, left ventricular to right atrial; FU, follow-up; TR, tricuspid regurgitation; VSD, ventricular septal defect. 
Table 2 Long-Term Outcome in 2 Groups of Patients

\begin{tabular}{lccccccccc}
\hline \hline & \multicolumn{3}{c}{ With LV-RA shunt } & & \multicolumn{3}{c}{ Without LV-RA shunt } & \multirow{2}{*}{ Total } \\
\cline { 2 - 3 } & Mild & Moderate & Severe & & Mild & Moderate & Severe & \\
\hline Spontaneous closure & 31 & 5 & 0 & & 20 & 5 & 1 & $62(27 \%)$ \\
Smaller VSD & 35 & 9 & 3 & & 31 & 7 & 4 & $89(38 \%)$ \\
Unchanged VSD & 29 & 9 & 0 & & 19 & 6 & 0 & $63(27 \%)$ \\
Surgery & 0 & 6 & 7 & & 0 & 3 & 2 & $18(8 \%)$ \\
\hline
\end{tabular}

Abbreviations see in Table 1.
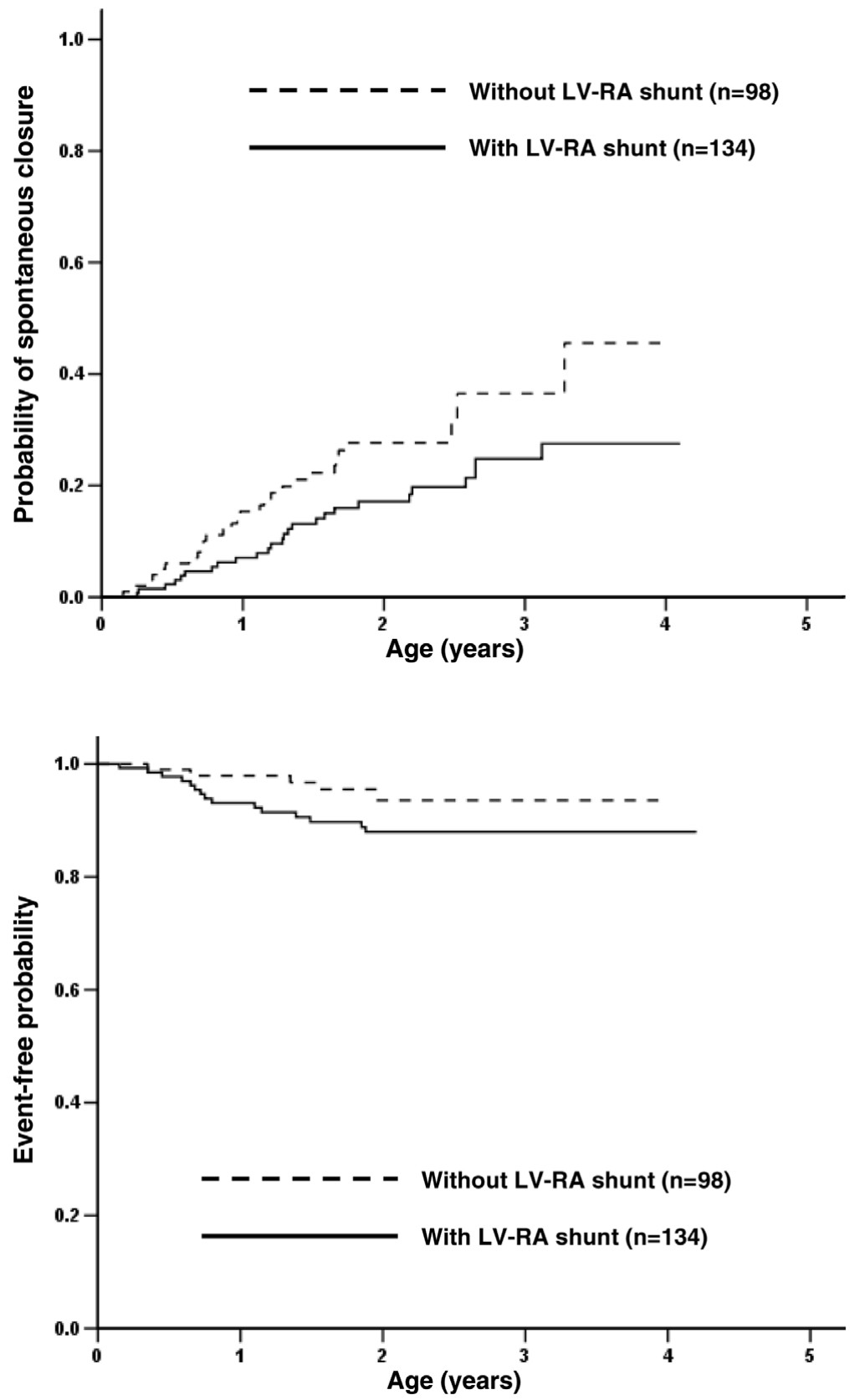

Fig 3. Probability of spontaneous closure with age in the 2 groups of patients. LV-RA, left ventricular to right atrial.
Fig 4. Event-free probability (no surgical repair of the defect) with age in the 2 groups of patients. LV$\mathrm{RA}$, left ventricular to right atrial. applicable to the rare data set. The event-free curves and probability of spontaneous closure were estimated by Kaplan-Meier analysis. Differences between 2 groups in the Kaplan-Meier analysis were evaluated by log-rank test. A p-value $<0.05$ was considered significant.

\section{Results}

All 232 patients had isolated perimembranous VSD and aneurysm. LV-RA shunt was identified in 134 patients (58\% of the total subjects). The remaining 98 patients did not have LV-RA shunts. The patients' characteristics are 
summarized in Table 1. There were no significant differences between groups in terms of sex, age at initial echocardiography, follow-up period, number of patients with TR, and initial VSD size. The follow-up outcomes are shown in Table2. In all, the proportions of spontaneous closure, smaller VSD, unchanged VSD, and surgery were $27 \%, 38 \%, 27 \%$, and $8 \%$ respectively.

Of the 134 patients with LV-RA shunt, 77 were males and 55 were females. The mean age at first color Doppler echocardiography was $3 \pm 3$ months. The mean age at detection of LV-RA shunt was $5 \pm 4$ months. Trivial LV-RA shunt was detected in 106 patients $(79 \%)$ and the remaining 28 patients $(21 \%)$ had small-to-moderate LV-RA shunts. In total, 95 patients had mild VSD, 29 had moderate VSD, and 10 had severe VSD; 128 patients had TR, which could be clearly differentiated from LV-RA shunt by the presence of the different positions and directions of the color jets. The mean follow-up period was $28 \pm 9$ months. Of the 10 patients with severe VSD, 3 had a smaller VSD and 7 underwent surgery. Of the 29 patients with moderate VSD, 5 had spontaneous closure, 9 had a smaller VSD, 9 had unchanged VSD with LV-RA shunt, and 6 underwent surgery. Of the 95 patients with mild VSD, 31 had spontaneous closure, 35 had a smaller VSD, and 29 had an unchanged shunt.

Of the 98 patients without LV-RA shunt, 65 were males and 33 were females. The mean age at first color Doppler echocardiography was $3 \pm 4$ months; 70 patients had mild VSD, 21 had moderate VSD, and 7 had severe VSD; 91 had TR. The mean follow-up period was $26 \pm 9$ months. Of the 7 patients with severe VSD, 1 had spontaneous closure, 4 had a smaller VSD, and 2 underwent surgery. Of the 21 patients with moderate VSD, 5 had spontaneous closure, 7 had a smaller VSD, 6 had an unchanged VSD, and 3 underwent surgery. Of the 70 patients with mild VSD, 20 had spontaneous closure, 31 had a smaller VSD, and 19 had an unchanged VSD.

The probability of spontaneous closure is shown in Fig 3: it was $28 \%$ in patients without LV-RA shunt, and $17 \%$ in those with LV-RA shunt at the age of 2 years. There was a significant difference between the 2 groups in the probability of spontaneous closure $(\mathrm{p}=0.039)$. Event-free probability (no surgical repair of the defect) with the endpoint defined as heart surgery is shown in Fig 4: it was $94 \%$ in patients without LV-RA shunt, and $88 \%$ in those with LV-RA shunt by the age of 2 years. The event-free probability was not significantly different between groups $(\mathrm{p}=0.129)$.

\section{Discussion}

LV-RA shunt, defined as echocardiographic evidence of high velocity turbulence moving directly from the LV into the RA, is reported to occur in $3-15 \%$ of patients with isolated perimembranous VSD $3,10-13$ In the present series, we defined LV-RA shunt in patients with isolated perimembranous VSD according to 2 criteria: the bank-shot sign and Doppler demonstration of high-velocity turbulence $(>4.0 \mathrm{~m} / \mathrm{s})$ moving from the $\mathrm{LV}$, through the VSD, directly into the RA2,3 Because of advances in color Doppler echocardiography, we were able to accurately demonstrate in vivo both the exact pathway of the shunt and the bank-shot sign. These new criteria may explain why $58 \%$ of the patients were found to have LV-RA shunt in this series.

Several types of LV-RA shunt have been proposed, according to findings from pathological-surgical examina- tion of the heart! ${ }^{17}, 18$ Although different classifications exist, essentially 2 major types have been identified: the infravalvular type caused by various tricuspid anomalies and the supravalvular type caused by a defect in the atrioventricular part of the interventricular septum. In the present series, the LV-RA shunts were the infravalvular type. Of note was the characteristic flow pattern (the bank-shot sign) on color Doppler echocardiography. To the best of our knowledge, this sign has not been described previously. We postulate that, in this type of LV-RA shunt, the septal aneurysm of the tricuspid valve acts as a partial or complete barrier to the VSD, and blood flow from the LV bifurcates into 2 jets: 1 through the residual defect in the aneurysm into the RV and the other through the defective septal leaflet into the RA. The bifurcation and splitting of the blood flow proximal to the septal aneurysm at the site of the VSD is demonstrated vividly on color flow maps. Using the cine-loop function may enhance the ability to detect this particular flow, which can be missed on real-time images because the flow with LV-RA shunt is often trivial. To exclude falsepositive results, the color gain must be set properly. Other evidence supporting the diagnosis of LV-RA shunt was the high-velocity jet going back to the RA. Appropriate use of color Doppler echocardiography greatly improves the diagnostic efficacy in assessment of LV-RA shunt.

The effect of LV-RA shunt on spontaneous closure has long been an important issue. In agreement with previous studies, approximately one-third of the present patients had spontaneous closure 3,19 However, there is a great difference between our study and previous reports with regard to spontaneous closure of a perimembranous VSD with LV-RA shunt $3,13 \mathrm{Wu}$ et al reported that no patients with LV-RA shunt had spontaneous closure of the perimembranous VSD during serial echocardiographic examinations since infan$\mathrm{cy}^{3}$, and Erolu et al reported only 1 spontaneous closure in a series of patients with LV-RA shunt ${ }^{13}$ In the present series, the probability of spontaneous closure was significantly higher in patients without LV-RA shunt than in those with LV-RA shunt, but although the presence of a shunt was associated with a lower probability of spontaneous closure, spontaneous closure was not rare. The difference between our findings and those of the previous reports may relate to the definition of trivial LV-RA shunt, which can not be identified easily using old-fashioned color Doppler echocardiography.

In this series, TR was often found in patients with or without LV-RA shunt. Coexistence of LV-RA shunt and TR is very common, so color flow images should be carefully examined to avoid the confusion. Two factors may account for the high incidence of TR in these patients with perimembranous VSD. One factor is associated with normal variation of the tricuspid valve. Rosenquist et al reported a relatively high incidence of normal variations in the tricuspid valve attachment to the membranous ventricular septum?20 The finding of a grossly perfect, but possibly microscopically imperfect, tricuspid valve, even in a healthy individual, may partly explain the high incidence of TR associated with perimembranous VSD. The other factor is associated with aneurysm formation. TR in some patients with aneurysm formation has been attributed to a distortion of the septal and anterior leaflets, resulting in significant shunt.21-24

Data on the natural history of LV-RA shunts in perimembranous VSD during infancy are limited. Wu et al reported that VSD with secondary LV-RA shunt is associated with a higher risk for infective endocarditis, but still had a low 
chance for late improvement and even closure? In the present series, the event-free probability was not significantly different in both groups. LV-RA shunt may not aggravate the hemodynamic changes in patients with mild VSD, but may contribute to congestive heart failure and the need for surgical intervention in patients with a large VSD. Our study is a preliminary report of the natural history of LVRA shunt in patients with perimembranous VSD during infancy.

\section{Study Limitations}

Several limitations need to be specified. This was not a perfect cohort study. The relatively small number of patients could have introduced certain bias in our results. The definition of the bank-shot sign may be affected by a certain degree of subjectivity involved in the analysis of the color flow. Thus, further studies are required to elucidate these factors.

In conclusion, LV-RA shunt is very common in pediatric patients with isolated perimembranous VSD. Perimembranous VSD with LV-RA shunt in infancy is associated with a lower chance of spontaneous closure. Color Doppler echocardiography can greatly improves the diagnostic efficacy and help us understand the possible mechanisms leading to this particular anomaly.

\section{Acknowledgments}

This study was supported by grants from Kaohsiung Veterans General Hospital (VGHKS 96-052) and Zuoying Armed Forces Hospital (9701).

\section{References}

1. Ramaciotti C, Keren A, Silverman NH. Importance of (perimembranous) ventricular septal aneurysm in the natural history of isolated perimembranous ventricular septal defect. Am J Cardiol 1986; 57: $268-272$.

2. Leung MP, Mok CK, Lo RN, Lau KC. An echocardiographic study of perimembranous ventricular septal defect with left ventricular to right atrial shunting. Br Heart J 1986; 55: 45-52.

3. Wu MH, Wu JM, Chang CI, Wang JK, Wu YN, Chien SC, et al. Implication of aneurysmal transformation in isolated perimembranous ventricular septal defect. Am J Cardiol 1993; 72: 596-601.

4. Wu MH, Chang CI, Wang JK, Lue HC. Characterization of aneurysmal transformation in perimembranous ventricular septal defects: An adhered anterior leaflet of tricuspid valve predisposes to the development of left ventricular-to-right atrial shunt. Int J Cardiol 1994; 47: $117-125$.

5. Wu MH, Wang JK, Lin MT, Wu ET, Lu FL, Chiu SN, et al. Ventricular septal defect with secondary left ventricular-to-right atrial shunt is associated with a higher risk for infective endocarditis and a lower late chance of closure. Pediatrics 2006; 117: e262-e267.
6. Stahaman M, Kaplan S, Helmsworth JA, Clark LC, Scott HW Jr. Syndrome of left ventricular-right atrial shunt resulting from high interventricular septal defect associated with defective septal leaflet of the tricuspid valve. Circulation 1995; 12: 813-818.

7. Kirby CK, Johnson J, Zinsser HF. Successful closure of a left ventricular-right atrial shunt. Ann Surg 1957; 145: 392-394.

8. Ellio LP, Gedgaudas E, Levy MJ. Edwards JE. The Roentgenologic Findings in left ventricular-right atrial communication. Am J Roentgenol 1965; 93: 304-314.

9. Braunwald E, Morrow AG. Ventriculo-right atrial communication; diagnosis by clinical, hemodynamic and angiographic methods. Am J Med 1960; 28: 913-920.

10. Wu MH, Lue HC, Wang JK, Hung CR. Color flow mapping in preimembranous ventricular septal defect with left ventricular-to-right atrial shunts. J Formosan Med Assoc 1989; 88: 38-42.

11. Helmcke F, de Souza A, Nanda NC, Villacosta I, Gatewood R Jr, Colvin E, et al. Two-dimensional and color Doppler assessment of ventricular septal defect of congenital origin. Am J Cardiol 1989; 63: $1112-1116$.

12. Ramaciotti C, Vetter JM, Bornemeier RA, Chin AJ. Prevalence, relation to spontaneous closure, and association of muscular ventricular septal defects. Am J Cardiol 1995; 75: 61-65.

13. Erolu AG, Oztunc F, Saltik L, Bakari S, Dedeolu S, Ahunbay G. Evolution of ventricular septal defect with special reference to spontaneous closure rate, subaortic ridge and aortic valve prolapse. Pediatr Cardiol 2003; 24: 31-35.

14. Miyatake K, Okamoto M, Kinoshita N, Izumi S, Owa M, Takao S, et al. Clinical applications of a new type of real-time two-dimensional Doppler flow imaging system. Am J Cardiol 1984; 54: 857-868.

15. Sahn DJ. Real-time two-dimensional Doppler echocardiographic flow mapping. Circulation 1985; 71: 849-853.

16. Sharif DS, Huhta JC, Marantz P, Hawkins HK, Yoon GY. Twodimensional echocardiographic determination of ventricular septal defect size: Correlation with autopsy. Am Heart J 1989; 117: 1333 1336.

17. Gerbode F, Hultgren H, Melrose D, Osborn J. Syndrome of left ventricular-right atrial shunt successful surgical repair of defect in five cases, with observation of bradycardia on closure. Ann Surg 1958; 148: $433-446$

18. Sakakibara S, Konno S. Left ventricular-right atrial communication. Ann Surg 1963; 158: 93-99.

19. Moe DG, Guntheroth WG. Spontaneous closure of uncomplicated ventricular septal defect. Am J Cardiol 1987; 60: 674-678.

20. Rosenquist GC, Sweeney LJ. Normal variations in tricuspid valve attachments to the membranous ventricular septum: A clue to the etiology of left ventricle-to-right atrial communication. Am Heart $J$ 1975; 89: $186-188$.

21. Hagler DJ, Squarcia U, Cabalka AK, Connolly HM, O'Leary PW. Mechanism of tricuspid regurgitation in paramembranous ventricular septal defect. J Am Soc Echocardiogr 2002; 15: 364-368.

22. Tandon R, Edwards JE. Aneurysmlike formations in relation to membranous ventricular septum. Circulation 1973; 47: 1089-1097.

23. Anderson RH, Lenox CC, Zuberbuhler JR. Mechanisms of closure of perimembranous ventricular septal defect. Am J Cardiol 1983; 52: 341-345.

24. Eshaghpour E, Kawai N, Linhart JW. Tricuspid insufficiency associated with aneurysm of the ventricular septum. Pediatrics 1978; 61: $586-592$. 